\title{
De nouveaux antibiotiques : oui mais pour quelles situations en réanimation ?
}

\author{
New Antibiotics: yes, but for which Situations in the Intensive Care Unit?
}

\author{
M Wolff \\ Reçu le 13 mars 2017; accepté le 14 mars 2017 \\ (C) SRLF et Lavoisier SAS 2017
}

Le relatif réinvestissement de l'industrie pharmaceutique dans le développement de nouvelles molécules antiinfectieuses est une excellente nouvelle au regard de l'émergence de la résistance bactérienne. L'isolement plus fréquent, notamment dans les services de réanimation, de bactéries hautement résistantes rend malheureusement possible « la fin du miracle », c'est-à-dire le retour d'un monde sans antibiotiques, comme le craignait Stuart Lévy voici déjà un quart de siècle [1]. Depuis quelques années pourtant, les espoirs renaissent avec l'arrivée de molécules innovantes dont certaines font l'objet d'une mise au point dans le présent numéro. D'éminents spécialistes permettent au lecteur d'avoir une idée claire et précise sur les propriétés antibactériennes, les caractéristiques pharmacologiques et pharmacodynamiques et l'efficacité clinique de molécules récentes ou récemment remises au goût du jour. À la lumière des données publiées, le réanimateur se sent pourtant parfois frustré et ceci pour deux raisons : d'une part, en accord avec les recommandations des autorités de régulation, le développement de nouvelles molécules porte initialement sur des infections peu graves avec une extrapolation non évidente pour les patients en situation critique. D'autre part, les posologies utilisées ne sont souvent pas adaptées à l'augmentation fréquente du volume de distribution observée chez ces patients [2] et peuvent, au moins en partie, expliquer certains échecs, notamment ceux de la tigécycline et du ceftobiprole au cours de pneumonies acquises sous ventilation mécanique.

Essentiellement pour des raisons épidémiologiques liées à la forte incidence des infections à staphylocoques dorés résistant à la méticilline (SARM) ou à entérocoques résistant à la vancomycine (ERV) en Amérique du Nord, les efforts de l'industrie ont d'abord porté sur les molécules actives sur ces

\footnotetext{
M Wolff $(\bowtie)$

Service de réanimation médicale et des maladies infectieuses, hôpital Bichat-Claude Bernard, 46, rue Henri-Huchard, F-75018 Paris, France

e-mail : michel.wolff@aphp.fr
}

bactéries. Ces efforts ont permis la mise sur le marché de molécules innovantes telles que les oxazolidinones (Zyvo$\mathrm{xid}^{\circledR}$ ) et les nouveaux glycopeptides ou lipopeptides (daptomycine). L'arrivée des céphalosporines dites « de $5^{\mathrm{e}}$ génération » (ceftaroline fosamil et ceftobiprole médocaril) constitue une véritable révolution puisque nous disposons, pour la première fois dans l'histoire de l'antibiothérapie, de $\beta$-lactamines actives sur les SARM, du fait de leur affinité conservé sur la PLP2a. Pour autant, les indications de ces molécules restent à préciser, d'autant qu'elles sont actuellement majoritairement utilisées en seconde ligne dans des bactériémies à SARM [3]. Pourtant, leur très bon profil de tolérance (celui des $\beta$-lactamines) et leur effet bactéricide rapide associé à un spectre large incluant des entérobactéries devraient en faire de très sérieux concurrents aux glyco/lipopeptides, y compris dans le traitement probabiliste lorsque le risque de SARM est jugé élevé.

Plus de 15 ans après son arrivée, la résistance au linézolide reste marginale et cette molécule a trouvé sa place dans le traitement des infections à staphylocoques résistant à la méticilline lorsqu'une bonne diffusion tissulaire est prioritairement recherchée. La limite principale à l'utilisation de cette molécule tient aux effets secondaires. Le tédizolide, dont la posologie quotidienne est six fois inférieure à celle du linézolide, semble mieux toléré, y compris au long cours. Une étude de phase 3 dans les pneumonies nosocomiales comparant linézolide vs tédizolide est en cours, avec un objectif d'inclusion de plus de 700 patients d'ici la fin de l'année 2018 (Tedizolid Phosphate [TR-701 FA] vs Linezolid for the Treatment of Nosocomial Pneumonia [MK1986-002]).

En France et dans beaucoup d'autres pays, les menaces actuelles et futures viennent surtout des bacilles à Gram négatif avec notamment l'émergence et la diffusion d'entérobactéries productrices de carbapénémases (EPC). Bien que plus limitée et confinée à certains services hospitaliers, les souches de Pseudomonas aeruginosa ou d'Acinetobacter baumannii hautement résistantes posent des problèmes 
thérapeutiques complexes. Dans ce contexte inquiétant, la place des nouvelles molécules ou d'anciennes « revigorées » se discute selon deux finalités.

La première est d'épargner les carbapénèmes pour le traitement d'infections à entérobactéries productrices de $\beta$ lactamases à spectre élargi (EBLSE). Même si ce concept d'épargne est controversé en raison de l'impact sur le microbiote intestinal des molécules utilisées en alternative, une restriction de l'exposition aux carbapénèmes reste souhaitable. L'excellente revue consacrée à l'association ceftolozane-tazobactam et à la témocilline permet au lecteur de bien comprendre les apports de ces deux molécules. Pour la première, les données collectées dans les études de phase 3 montrent que l'on peut être serein en l'utilisant dans des infections à $E$. Coli BLSE CTX-M, mécanisme très largement prédominant en France. Là encore, les études de phase 3 ont concerné des infections urinaires et abdominales avec peu de pertinence pour les malades de réanimation et une posologie peu adaptée à ces patients $(1 \mathrm{~g} / 0,5 \mathrm{~g}$ toutes les $8 \mathrm{~h})$. Il sera très intéressant de regarder en détail les résultats concernant les patients avec EBLSE dans l'étude en cours sur les pneumonies nosocomiales comparant ceftolozanetazobactam $(2 \mathrm{~g} / 1 \mathrm{~g}$ toutes les $8 \mathrm{~h})$ vs méropénème ( $1 \mathrm{~g}$ toutes les 8 h). Cette étude est prévue pour inclure 726 patients (Safety and Efficacy Study of Ceftolozane/Tazobactam to Treat Ventilated Nosocomial Pneumonia [MK-7625A-008] [ASPECT-NP]).

L'épargne des carbapénèmes se fait en principe après documentation mais la modification très précoce du microbiote par les carbapénèmes peut aussi faire discuter une utilisation en traitement probabiliste dans des conditions très strictes et prenant en compte les facteurs de risque d'EBLSE. Le spectre large de ceftolozane-tazobactam pourrait autoriser cette utilisation en probabiliste ce qui n'est pas le cas pour la témocilline, non active sur les bacilles à Gram négatif aérobies stricts. En revanche, la témocilline est active à la fois sur les EBLSE et les entérobactéries hyperproductrices de céphalosporinase. Malgré son ancienneté, les données cliniques concernant la témocilline restent assez limitées, voire quasi inexistantes si l'on s'intéresse aux infections sévères liées aux entérobactéries précédemment citées. On peut espérer que des études sur cette molécule seront conduites dans un proche avenir et cibleront les malades de réanimation.

La seconde utilisation possible des nouvelles molécules concerne la prise en charge des bacilles à Gram négatif hautement résistants. Si ceftolozane-tazobactam n'est pas active sur les EPC, cette molécule est actuellement la plus puissante sur $P$. aeruginosa. Cette supériorité sur cette bactérie par rapport aux autres antibiotiques est liée au fait que ceftolozane-tazobactam n'est pas un substrat pour les principales pompes à efflux et qu'elle reste active sur un pourcentage notable de souches très résistantes qui associent plu- sieurs mécanismes (céphalosporinase, efflux et mutation sur la porine $\mathrm{D} 2$ ). Très récemment, une équipe espagnole a rapporté des résultats intéressants sur 12 malades ayant reçu cette association en traitement de sauvetage pour une infection à $P$. aeruginosa résistante à toutes les autres $\beta$ lactamines et à la ciprofloxacine (dont une moitié de pneumonies). Huit des 12 patients ont guéri sans rechute mais dans deux cas, une souche résistante a émergé [4].

Le grand défi des prochaines années sera celui du traitement des infections à EPC dont l'incidence est appelée à augmenter surtout pour les classes B (notamment les souches « NDM ») et D (OXA-48). Parmi les associations avec inhibiteurs de $\beta$-lactamase, seules celles contenant l'avibactam sont potentiellement utiles. Les premières études cliniques avec ceftazidime-avibactam sur des infections à EPC des groupes A (KPC) et D sont plutôt encourageantes [5,6] mais malheureusement on voit déjà apparaître des souches résistantes avec de nouveaux mécanismes récemment décrits tels que bla KPC-3 [7]. L'association de l'avibactam (inhibiteur de BLSE) avec l'aztréonam (plus stable vis-à-vis de NDM et VIM) pourrait être utilisée pour traiter des infections à EPC du groupe B. Cette association n'étant pas encore disponible, des auteurs ont proposé l'administration combinée de ceftazidime-avibactam + aztréonam [8]. Dans les conditions actuelles, le clinicien, confronté à une infection par EPC, doit cependant recourir à des associations plus ou moins complexes et dont les interactions ne sont pas toujours bien connues. Pour les classes A et D, il convient d'utiliser une association, au mieux incluant quand même un carbapénème [9] avec la colistine ou la tygécycline. Cette dernière trouve toute sa place ici, comme cela est très bien expliqué dans l'article qui lui est consacré dans ce numéro. Une activité in vitro deux à quatre fois supérieure et des profils pharmacocinétiques et de tolérance plus favorables pourraient donner un certain avantage à l'eravacycline, une nouvelle cycline de synthèse [10].

En conclusion, le pipeline des antibiotiques n'est plus à sec. Pour autant, les bactéries hautement résistantes sont et seront un défi majeur. Un usage raisonné des nouvelles molécules contribuera à les préserver le plus longtemps possible au bénéfice de patients très souvent atteints de multiples comorbidités ou immunodéprimés.

Liens d'intérêts : Le Dr Wolff déclare des participations à un board pour MSD et Sanofi, et des interventions comme orateur dans des symposiums.

\section{Références}

1. Levy SB, (1992) The Antibiotic Paradox: How Miracle Drugs Are Destroying the Miracle. New York, Plenum, 279 pp. 
2. Roberts JA, Taccone FS, Lipman J, (2016) Understanding PKPD. Intensive Care Med 42: 1797-1800

3. Zasowski EJ, Trinh TD, Claeys KC, Casapao AM, Sabagha N, Lagnf AM, Klinker KP, Davis SL, Rybak MJ, (2017) Multicenter observational study of Ceftaroline Fosamil for methicillinresistant Staphylococcus aureus bloodstream infections. Antimicrob Agents Chemother 61: pii: e02015-16

4. Castón JJ, De la Torre Á, Ruiz-Camps I, Sorlí ML, Torres V, Torre-Cisneros J, (2017) Salvage therapy with ceftolozanetazobactam for multidrug resistant $P$. aeruginosa infections. Antimicrob Agents Chemother 61: pii: e02136-16

5. Shields RK, Potoski BA, Haidar G, Hao B, Doi Y, Chen L, Press EG, Kreiswirth BN, Clancy CJ, Nguyen MH, (2016) Clinical outcomes, drug toxicity, and emergence of ceftazidimeavibactam resistance among patients treated for carbapenemresistant Enterobacteriaceae infections. Clin Infect Dis 63: 1615-1618

6. Temkin E, Torre-Cisneros J, Beovic B, Benito N, Giannella M, Gilarranz R, Jeremiah C, Loeches B, Machuca I, Jiménez-Martín MJ, Martínez JA, Mora-Rillo M, Navas E, Osthoff M, Pozo JC, Ramos Ramos JC, Rodriguez M, Sánchez-García M, Viale P, Wolff M, Carmeli Y, (2017) Ceftazidime-avibactam as salvage therapy for infections caused by carbapenem-resistant organisms. Antimicrob Agents Chemother 24: pii: e01964-16

7. Haidar G, Clancy CJ, Shields RK, Hao B, Cheng S, Nguyen MH, (2017) Mutations in blaKPC-3 that confer ceftazidime-avibactam resistance encode novel KPC-3 variants that function as extended-spectrum $\beta$-lactamases. Antimicrob Agents Chemother 21: pii: AAC.02534-16

8. Marshall S, Hujer AM, Rojas LJ, Papp-Wallace KM, Humphries RM, Spellberg B, Hujer KM, Marshall EK, Rudin SD, Perez F, Wilson BM, Wasserman RB, Chikowski L, Paterson DL, Vila AJ, van Duin D, Kreiswirth BN, Chambers HF, Fowler VG Jr, Jacobs MR, Pulse ME, Weiss WJ, Bonomo RA, (2017) Can ceftazidime/ avibactam and aztreonam overcome $\beta$-lactam resistance conferred by metallo- $\beta$-lactamases in Enterobacteriaceae? Antimicrob Agents Chemother [in press]

9. Tzouvelekis LS, Markogiannakis A, Piperaki E, Souli M, Daikos ML, (2014) Treating infections caused by carbapenemaseproducing Enterobacteriaceae. Clin Microbiol Infect 20: 862872

10. Livermore D, Mushtaq S, Woodford N, (2016) In vitro activity of eravacycline against carbapenem-resistant enterobacteriaceae and Acinetobacter baumannii. Antimicrob Agents Chemother 60: 3840-3844 